\title{
On the Purification of Nash Equilibria of
}

\author{
Large Games*
}

\author{
Guilherme Carmona ${ }^{\dagger}$ \\ Universidade Nova de Lisboa
}

September 29, 2003

\begin{abstract}
We consider Salim Rashid's asymptotic version of David Schmeidler's theorem on the purification of Nash equilibria. We show that, in contrast to what is stated, players' payoff functions have to be selected from an equicontinuous family in order for Rashid's theorem to

${ }^{*}$ I wish to thank Mário Páscoa and Myrna Wooders for very helpful comments. All remaining errors are, of course, mine.

${ }^{\dagger}$ Address: Universidade Nova de Lisboa, Faculdade de Economia, Campus de Campolide, 1099-032 Lisboa, Portugal; Phone: (351) 21380 1671; Fax: (351) 2138860 73;
\end{abstract} email: gcarmona@fe.unl.pt. 
hold. That is, a bound on the diversity of payoffs is needed in order for such asymptotic result to be valid.

Keywords: Nash Equilibrium; Purification; Asymptotic Results; Equicontinuity.

JEL classification: C72.

\section{Introduction}

A special class of games are those in which each player payoff depends only his choice and on the average choice of the others. This class of games is interesting because all Nash equilibria of any such game can be purified, whenever the set of players is described by a non-atomic measure space (see Schmeidler [6]). Salim Rashid [5] stated an asymptotic version of Schmeidler's theorem, according to which all Nash equilibria of sufficiently large games of the same class can be approximately purified. Asymptotic results are important because, after all, real-world games have finitely many players.

The goal of this note is to correct the statement of Rashid's theorem. We will show by an example that, as stated in his paper, the theorem is incorrect. In our example, the crucial feature is that we use payoff functions 
belonging to a family that is not equicontinuous. In fact, we show that Rashid's theorem holds once an equicontinuity assumption is added. This result stresses the importance of equicontinuity assumptions for asymptotic results on Nash equilibria. ${ }^{1}$

\section{Approximate Purification of Nash Equilib- ria}

In the class of normal-form games we consider, all players have the same strategy space: the unit simplex $s_{m}$ on $\mathbb{R}^{m}, m>1$. Since the focus is on a property that depends on the number of players, we will index any game by the number of its players. Thus, $G_{n}$ is a normal-form game in which the set of player is $\{1, \ldots, n\}$, and each has $s_{m}$ as her choice set. To each player $t$, we associate $m$ continuous functions $V_{t k}: s_{m} \rightarrow \mathbb{R}, 1 \leq k \leq m$; then player t's payoff function is

$$
U_{t}\left(x_{1}, \ldots, x_{n}\right)=x_{t 1} V_{t 1}\left(\sum_{j \neq t} \frac{x_{j}}{n}\right)+\ldots+x_{t m} V_{t m}\left(\sum_{j \neq t} \frac{x_{j}}{n}\right),
$$

\footnotetext{
${ }^{1}$ See Cartwright and Wooders [1], Hildenbrand [2] and Páscoa [4] among others for related results that also require equicontinuity assumptions.
} 
for all $\left(x_{1}, \ldots, x_{n}\right)$. Thus, $G_{n}$ is described by the vector $\left(V_{t k}\right)_{t, k}$. Also, if $\mathcal{U}$ denotes the space of all continuous, real-valued functions on $s_{m}$, then a game can be thought of as a subset of $\mathcal{U}$. For any subset $K$ of $\mathcal{U}$, we write $G_{n} \subseteq K$ whenever $V_{t k} \in K$ for all $1 \leq t \leq n$ and $1 \leq k \leq m$.

For any $\varepsilon \geq 0$, we say that $x^{*}=\left(x_{1}^{*}, \ldots, x_{n}^{*}\right)$ is an $\varepsilon$-equilibrium of a game $G_{n}$ if, for all $t \in\{1, \ldots, n\}$,

$$
U_{t}\left(x^{*}\right) \geq U_{t}\left(x_{t}, x_{-t}^{*}\right)-\varepsilon \text { for all } x_{t} \in s_{m} .
$$

Thus, in an $\varepsilon$-equilibrium, all players are close to their optimum by choosing according to $x^{*}$. A strategy $x^{*}$ is a Nash equilibrium of $G_{n}$ if $x^{*}$ is an $\varepsilon$-equilibrium of $G_{n}$ for $\varepsilon=0$.

Let $K$ be a subset of $\mathcal{U}$. We say that $K$ is equicontinuous (or, that the family $K$ of functions is equicontinuous) if for all $\eta>0$ there exists a $\delta>0$ such that

$$
|V(x)-V(y)|<\eta
$$

whenever $\|x-y\|<\delta, x, y \in s_{m}$ and $V \in K$ (see Rudin [7, p. 156]). In our framework, equicontinuity can be interpreted as placing "a bound on the diversity of payoffs" (see Khan, Rath and Sun [3]).

For any $\varepsilon>0$ and $m, n \in \mathbb{N}$, we say that a game $G_{n}$ satisfies condition $P(\varepsilon, m, n)$ if for any Nash equilibrium $x^{*}$ of $G_{n}$ there exists an $\varepsilon$-equilibrium 
$x^{* *}$ of $G_{n}$ satisfying $\left|U_{t}\left(x^{*}\right)-U_{t}\left(x^{* *}\right)\right|<\varepsilon$ for all $t \in\{1, \ldots, n\}$ and at least $n-m$ players use a pure strategy. We interpret this condition as saying that all Nash equilibrium can be approximately purified: both the gains from deviations and the number of player not playing a pure strategy can be made small.

Theorem 1 Let $K$ be an equicontinuous subset of $\mathcal{U}$. Then, for all $\varepsilon>0$ and all $m \in \mathbb{N}$ there exists $N \in \mathbb{N}$ such that $n \geq N$ and $G_{n} \subseteq K$ implies that $G_{n}$ satisfies condition $P(\varepsilon, m, n)$.

This theorem can be proven using Rashid [5]'s elegant proof, after we use the equicontinuity of $K$ to find $\delta>0$ such that $|V(x)-V(y)|<\varepsilon / 2$ whenever $\|x-y\|<\delta, x, y \in s_{m}$ and $V \in K$. Only then can Rashid's observation (1) be used to show that $\left|U_{t}\left(x^{*}\right)-U_{t}\left(x^{* *}\right)\right|<\varepsilon$ and to show that if $x^{* *}$ is a Nash equilibrium in the game $G=\left(V_{t k}\right)_{t, k}$ then $x^{* *}$ is an $\varepsilon$-equilibrium in the game where players payoff functions are $U_{t}\left(x_{1}, \ldots, x_{n}\right)=x_{t 1} V_{t 1}\left(\sum_{j=1}^{n} \frac{x_{j}}{n}\right)+$ $\ldots+x_{t m} V_{t m}\left(\sum_{j=1}^{n} \frac{x_{j}}{n}\right)$, for all $\left(x_{1}, \ldots, x_{n}\right)$.

The importance of the equicontinuity assumption for the above result is illustrated by the following example. The example shows that without it, the above theorem is false. 
We will show that there exists an $\varepsilon>0$ and $m \in \mathbb{N}$ such that for all $N \in \mathbb{N}$ there exists a game $G_{n}$ with $n \geq N$ in which condition $P(\varepsilon, m, n)$ does not hold. We let $\varepsilon=1$ and $m=2$ (i.e., there are two actions).

Let $N \in \mathbb{N}$ be given. Define $n=N+10$ and $G_{n}$ as follows:

$$
\begin{aligned}
& V_{t 1}\left(\sum_{j \neq t} \frac{x_{j 1}}{n}\right)=\alpha \frac{n}{n-1} \sum_{j \neq t} \frac{x_{j 1}}{n} \text { and } \\
& V_{t 2}\left(\sum_{j \neq t} \frac{x_{j 1}}{n}\right)=\alpha\left(1-\frac{n}{n-1} \sum_{j \neq t} \frac{x_{j 1}}{n}\right) .
\end{aligned}
$$

for all $1 \leq t \leq n$ and where $\alpha>n$.

We claim that not all equilibria of $G_{n}$ can be approximately purified. Define $x_{t 1}^{*}=1 / 2$ for all $1 \leq t \leq n$; clearly, $x^{*}$ is a Nash equilibrium of $G_{n}$. We will show that if $x^{* *}$ is a strategy in $G_{n}$ satisfying $\left|U_{t}\left(x^{*}\right)-U_{t}\left(x^{* *}\right)\right|<1$ for all $t \in\{1, \ldots, n\}$ and at least $n-2$ players use a pure strategy, then $x^{* *}$ is not an 1-equilibrium. Clearly, this will establish our claim.

Note first that there is a player $t$ with $x_{t 1}^{* *}=1$. We prove this claim by contradiction: suppose that there is no player $t$ satisfying $x_{t 1}^{* *}=1$. Then consider a player $i$ such that $x_{i 1}^{* *}=0$ (since $n-2>0$ players play pure strategies, such $i$ exists). We have that $U_{i}\left(x^{*}\right)=\alpha / 2$ and $U_{i}\left(x^{* *}\right)=\alpha\left(1-\frac{n}{n-1} \sum_{j \neq i} \frac{x_{j 1}^{* *}}{n}\right)$. Thus,

$$
\left|\alpha\left(1-\frac{n}{n-1} \sum_{j \neq i} \frac{x_{j 1}^{* *}}{n}\right)-\frac{\alpha}{2}\right|<1,
$$


which implies that

$$
\left|\sum_{j \neq i} \frac{x_{j 1}^{* *}}{n-1}-\frac{1}{2}\right|<\frac{1}{\alpha}<\frac{1}{10} .
$$

This in turn implies that

$$
\sum_{j \neq i} \frac{x_{j 1}^{* *}}{n-1}>\frac{1}{2}-\frac{1}{10}=\frac{4}{10}
$$

On the other hand, since $n-2$ players play $x_{j 1}^{* *}=0$, we obtain

$$
\sum_{j \neq i} \frac{x_{j 1}^{* *}}{n-1} \leq \frac{2}{n-1}=\frac{2}{N+9} \leq \frac{2}{10}
$$

which is a contradiction.

Similarly, we can show that at least one player $\tilde{t}$ plays $x_{\tilde{t} 1}^{* *}=0$. If $t$ is such that $x_{t 1}^{* *}=1$, we obtain that

$$
\sum_{j \neq \tilde{t}} \frac{x_{j 1}^{* *}}{n-1}-\sum_{j \neq t} \frac{x_{j 1}^{* *}}{n-1}=\frac{x_{t 1}^{* *}}{n-1}-\frac{x_{\tilde{t} 1}^{* *}}{n-1}=\frac{1}{n-1}
$$

Thus, if $\tilde{t}$ is optimizing by choosing $x_{\tilde{t} 1}^{* *}=0$, then it must be that $\sum_{j \neq \tilde{t}} \frac{x_{j 1}^{* *}}{n-1} \leq$ $\frac{1}{2}$, which in turn implies that $\sum_{j \neq t} \frac{x_{j 1}^{* *}}{n-1}<\frac{1}{2}$ and so that player $t$ is not optimizing. Similarly, if player $t$ is optimizing, then player $\tilde{t}$ is not. Also, we can choose one of them, denoted $\bar{t}$, such that $\bar{t}$ is not optimizing and

$$
\left|\sum_{j \neq \bar{t}} \frac{x_{j 1}^{* *}}{n-1}-\frac{1}{2}\right| \geq \frac{1}{2(n-1)}
$$


For concreteness, say that $\bar{t}=t$ (the remaining case is precisely analogous). Then, it follows that

$$
1-2 \sum_{j \neq t} \frac{x_{j 1}^{* *}}{n-1} \geq \frac{1}{n-1}
$$

This will be used to show that $x^{* *}$ is not an 1 -equilibrium, which will complete our example.

We have then that $U_{t}\left(x^{* *}\right)=\alpha \sum_{j \neq t} \frac{x_{j 1}^{* *}}{n-1}$, while if he where to choose $x_{t 1}=0$, he would get $\alpha\left(1-\sum_{j \neq t} \frac{x_{j 1}^{* *}}{n-1}\right)$. The difference of payoffs is

$$
\alpha\left(1-\sum_{j \neq t} \frac{x_{j 1}^{* *}}{n-1}\right)-\alpha \sum_{j \neq t} \frac{x_{j 1}^{* *}}{n-1}=\alpha\left(1-2 \sum_{j \neq t} \frac{x_{j 1}^{* *}}{n-1}\right) \geq \frac{\alpha}{n-1}>1
$$

Hence, $x^{* *}$ is not an 1 -equilibrium.

We conclude with a remark on our example. The modulus of continuity

$\omega(\delta)$ of $V_{t k}$ is $\frac{n \delta}{(n-1) \alpha}>\frac{n^{2} \delta}{n-1}$. This number converges to infinity with $n$, which shows that the family of payoff functions we used in the example is not equicontinuous. This account for the failure of the conclusion of Theorem 1 .

\section{References}

[1] Cartwright, E. and M. Wooders, 2003, On the Existence of an Equilibrium in Pure Strategies in Games with Many Players, mimeo, University 
of Warwick.

[2] Hildenbrand, W., 1974, Core and Equilibria of a Large Economy (Princeton University Press, Princeton).

[3] Khan, M., K. Rath and Y. Sun, 1997, On the Existence of Pure Strategy Equilibria in Games with a Continuum of Players, Journal of Economic Theory $76,13-46$.

[4] Páscoa, M., 1993, Approximate Equilibria in Pure Strategies for Nonatomic Games, Journal of Mathematical Economics 22, 223-241.

[5] Rashid, S., 1983, Equilibrium Points of Non-atomic Games: Asymptotic Results, Economics Letters 12, 7-10.

[6] Schmeidler, D., 1973, Equilibrium Points of Non-atomic Games, Journal of Statistical Physics 4, 295-300.

[7] Rudin, W., 1976, Principles of Mathematical Analysis (McGraw-Hill, Singapore). 